\title{
Tecnologías actuales y emergentes para la identificación animal y su aplicación en la trazabilidad animal ${ }^{\#}$
}

\author{
Current and emergent technologies for animal identification and their use in animal traceability \\ R Felmer*, R Chávez, A Catrileo, C Rojas \\ Instituto de Investigaciones Agropecuarias, INIA-Carillanca, Temuco, Chile
}

\begin{abstract}
SUMMARY
The use of labels for the identification of live animals and its products have been practiced for over 2000 years, being extensively used in Europe with the appearance of the first epidemic diseases. At present, the advances on crops and livestock genetic engineering, the appearance of new diseases related with food consumption (i.e. mad cow disease, E. coli 0157, etc.), and the discovery of contaminants in the food chain have produced great concern among the consumers. This is why it is so important that producers provide consumers with a guarantee on the product quality, specially since they now demand to know origin and quality of the food. In international markets, new procedures based on the concept "from gate to plate" have been implemented through the food chain, in order to bring out trademarks and certified processes which guarantee the quality of the food that goes out in the market. As a result of this new consumer demand, the "traceability concept" in food has been gradually incorporated in our vocabulary and it is now one of the main concerns of the food industry. The traceability systems used to identify animals, monitor their movements and trace their products have considerably evolved over the last years. These systems must be convenient, easy to read, durable, and harmless. Many devices have been used pursuing this objective, such as tattoos, ear-tags, electronic chips, iris and retina identification and more recently, DNA fingerprinting and molecular markers. It has become important to harmonise approved systems in order to provide a better guarantee and to facilitate international trade of animals and their products. Today, traceability is an esssential requirement in terms of exporting high-value products to the global food markets providing the producer with an opportunity to reach better prices for a differentiated product. Thus, many meat industries have turned this concern in a great commercial opportunity.
\end{abstract}

Palabras clave: trazabilidad, microsatélites, identificación individual.

Key words: traceability, microsatellites, individual identification.

\section{INTRODUCCION}

Desde finales de la década de los 80 , los consumidores, especialmente europeos y norteamericanos, han visto con preocupación la aparición de una serie de crisis agroalimentarias originadas por el alcance y amplia difusión de patologías, como la encefalopatía espongiforme bovina (EEB o "mal de las vacas locas") y ovina ("scrapie"), la fiebre aftosa en bovinos, ovinos, caprinos y porcinos, los reiterados focos de peste porcina clásica (PPC), así como la detección de productos no permitidos o peligrosos en el ganado y en alimentos de origen animal, tales como hormonas y anabolizantes (clembuterol), antibióticos, pesticidas, contaminación con plomo, o el envenenamiento de la carne de hamburguesas por E. coli cepa $\mathrm{O} 157$ enterohemorrágica (EHEC). Todo esto ha causado gran alarma en muchos

Aceptado: 21.03.2006.

\# Financiado en parte por proyectos FDI-CORFO 03C9AT-01 y 02C8AD-10.

* Laboratorio de Biotecnología Animal, Unidad de Biotecnología, INIA-Carillanca. Camino Cajón Vilcún s/n Km. 10. Casilla 58D, Temuco. Fono: 45-215706; Fax: 45-216112; rfelmer@inia.cl países y resalta al mismo tiempo la facilidad con que actualmente se extienden estos problemas y la complejidad de su control y prevención (Pettitt 2001). Para controlar estos riesgos a la salud humana en la cadena de alimentos y así asegurar la confianza del consumidor, las autoridades públicas necesitan información que les permita establecer rápidamente la fuente de infección o contaminación de los productos alimenticios. Surge así la imperiosa necesidad de establecer políticas de identificación y seguimiento individual de los animales, mediante la aplicación de nuevas tecnologías adaptadas a la modernización y globalización de los intercambios comerciales actuales, con lo que empieza a tomar notoriedad el término trazabilidad.

En forma general, la trazabilidad es un conjunto de acciones, medidas y procedimientos técnicos que permite identificar y registrar cada producto desde su origen hasta el final de la cadena de comercialización. Así, la trazabilidad faculta rastrear la cadena de producción y otorga a los productores la posibilidad de colocar sus productos en mercados específicos más rentables, que exigen la certeza del origen y de las distintas etapas del proceso productivo. En el caso particular de los animales para consumo humano, esto significa seguir su procesamiento en toda la cadena, desde el nacimiento del 
animal, pasando por la crianza y prácticas ganaderas en el predio, transporte, faenamiento en matadero, hasta su puesta a la venta al detalle. En todo este proceso, se deben asegurar la identidad y calidad del producto. De esta forma, ante cualquier duda o problema, se puede rastrear su paso por la cadena productiva hasta el mismo predio donde nació el animal, verificando la forma en que fue alimentado y qué vacunas o medicamentos recibió, dónde y cómo fue faenado, etc.

Dado el crecimiento en el intercambio global y las preocupaciones del consumidor por la seguridad alimentaria, hoy en día se requieren sistemas más rápidos y efectivos de identificación y verificación animal que los clásicos crotales o registros manuales, utilizados por décadas en programas de erradicación de enfermedades en varios países, pues una de las principales consideraciones para un sistema de identificación y verificación animal es que permita la adquisición de la información en forma rápida y segura. Además, debe ser seguro contra fraudes y de un costo razonable para permitir la identificación de toda la masa ganadera, sin mayores perjuicios económicos para el productor. Los beneficios de tal sistema son para todos los participantes de la cadena alimenticia, desde el consumidor hasta la industria de alimentos, ya que este sistema contribuye a la seguridad alimentaria y a la certificación de calidad; además, ayuda a limitar la distribución de enfermedades en los animales, disminuyendo las pérdidas al productor debido a la presencia de las mismas.

\section{TECNOLOGIAS ACTUALES Y EMERGENTES PARA LA IDENTIFICACION ANIMAL}

Uno de los principales requisitos en la trazabilidad de animales y sus subproductos es la implementación de un sistema de identificación animal. Existe una serie de tecnologías actualmente en aplicación o desarrollo para la identificación animal y sus productos. Dentro de los métodos clásicos, se pueden destacar el marcaje de cola, tatuajes, marcas de fuego, marcas de frío, crotales o caravanas, que pueden ser de metal o plástico y los chips electrónicos. También existen los métodos biométricos tales como la huella nasal, imágenes digitales de retina e iris y la huella genética de ADN ("DNA fingerprinting"). Dentro de los métodos no biométricos, los más utilizados han sido el crotal o caravana. Los implantes de chips electrónicos han sido rechazados por las autoridades en EE.UU. debido al riesgo de que estos sistemas puedan migrar y entrar a la cadena de alimento. Por otro lado, el marcaje con fuego está prohibido en países del Reino Unido, debido a las fuertes medidas de protección y cuidados del bienestar animal.

Antes de describir los diferentes sistemas de identificación animal y aquellos que pueden aparecer en el futuro, es necesario considerar algunos aspectos clave que determinan la conveniencia en la utilización de uno u otro sistema.
Conveniencia y facilidad de uso. Aunque los productores capacitados y médicos veterinarios de terreno sean profesionales bien entrenados, los sistemas de identificación animal deben considerar la facilidad en su aplicación debido al gran número de animales que eventualmente necesitan ser identificados. Este número puede superar las 1.000 cabezas de ganado en algunos predios de Chile, mientras que en otros países la cifra puede elevarse incluso por sobre las 10.000 cabezas de ganado, lo que supone un problema de logística mayor.

Facilidad para leer. Se debe tener en cuenta que la identificación animal sea de fácil lectura en cualquier etapa de la cadena de producción, lo que es de vital importancia. Esto varía dependiendo del sistema de producción y de la habilidad para adoptar y acceder a la tecnología (Brappat 1996). Además, la exactitud de la lectura y la transmisión de los datos contenidos en el elemento de identificación también son aspectos claves. El avance tecnológico permite sistemas de caracteres alfanuméricos y códigos de barra combinados en un único elemento, lo cual reduce considerablemente los errores en la lectura, registro y transmisión de la información (Caja y col 1996).

Durabilidad. Otro punto a considerar es la durabilidad de los datos en la etiqueta, ya sea que estos sean impresos utilizando caracteres alfanuméricos, códigos de barra o sistemas electrónicos. A esto se agregan las características de resistencia a sustancias fisiológicas (sangre, sudoración), resistencia a las condiciones climáticas (temperatura, radiación solar) así como también a los materiales utilizados en la elaboración de los sistemas (Comisión de la Comunidad Europea 1997). Otro punto importante es su resistencia al desprendimiento del animal en lugares de pastoreo con matorrales en alta densidad.

Inocuidad. Finalmente, los elementos de identificación deben ser bien tolerados por el animal sin producir efectos adversos en su salud ni tampoco deben constituir un riesgo para la salud pública, a través del contacto, contaminación o por cualquier otra ruta (Comisión de la Comunidad Europea 1997).

1.1 METODOS NO-BIOMETRICOS DE IDENTIFICACION ANIMAL

Tatuajes. Este método de identificación permanente consiste en tatuar la combinación de letras y números en la oreja del animal con tinta indeleble. El equipo para tatuar consiste en una herramienta semejante a unos alicates, con un juego de letras y números, que tienen una serie de proyecciones afiladas como agujas que permiten perforar la oreja. Una vez perforada la oreja, se frota tinta indeleble sobre las diminutas perforaciones de manera que, al cicatrizar la herida, el tatuaje queda de 
forma permanente. Si bien esta es una manera eficaz de identificar permanentemente a un animal, su principal desventaja es su poca visibilidad desde distancias lejanas, por lo que no es recomendable como único sistema de identificación. Por otro lado, dentro de un esquema de trazabilidad, este sistema es insuficiente para la identificación animal, pues todos los animales del rebaño llevan la misma marca. Además de lo anterior, este sistema no es reconocido internacionalmente como una forma válida de identificación animal.

Crotales, aretes o caravanas. Es la forma de identificación más comúnmente usada. Los aretes plásticos y flexibles son fáciles de colocar y pocas veces se pierden. Se colocan con una herramienta especial para perforar la oreja, con un botón con tallo rígido o un botón para el revés de la oreja, que permite ver el número de identificación en la parte posterior de la oreja del animal. Los aretes son económicos, se pueden conseguir de diferentes colores dependiendo de la exigencia de cada país y pueden ser numerados. También se encuentran en formatos metálicos, con códigos de barra o con chips electrónicos que facilitan la lectura, transmisión y registro de datos. Aunque los aretes metálicos son más baratos y más resistentes a las pérdidas que los de plástico, una mala aplicación de estos puede inducir infecciones en la oreja del animal (Stanford y col 2001). La normativa chilena recientemente implementada, exige la utilización de dos dispositivos de identificación individual oficial (DIIO). Uno de ellos es un dispositivo visual de tipo paleta, con doble paleta (macho-hembra), de material plástico, color amarillo y con números y letras en color negro impresas en láser, tinta o la combinación de ambas (figura 1). La identificación de Chile como país de origen es a través de la sigla $C L$, seguida de la identificación del Servicio Agrícola y Ganadero (a través de las letras SAG) y la identificación del animal a través de un número correlativo único de 9 dígitos (para más detalles dirigirse a www.trazabilidad.sag.gob.cl). El segundo componente es un dispositivo visual tipo botón, que debe contener el número de identificación correspondiente; opcionalmente, este segundo componente puede ser remplazado por un dispositivo (botón o bolo ruminal) con radiofrecuencia (RFID). Si la persona opta por utilizar un dispositivo electrónico, éste deberá cumplir con las Normas de Fabricación ISO 11784 e ISO 11785.

Métodos de identificación electrónica (IDE). La necesidad de disponer de métodos de identificación animal que faciliten la trazabilidad de sus productos y que puedan ser usados globalmente despertó el interés por la identificación electrónica (IDE) mediante dispositivos pasivos de radiofrecuencia que utilizan radiaciones electromagnéticas no ionizantes. Estos sistemas están constituidos por dispositivos electrónicos pasivos de pequeño tamaño llamados transponders (figura $2 \mathrm{~A}$ ) que son sondea-

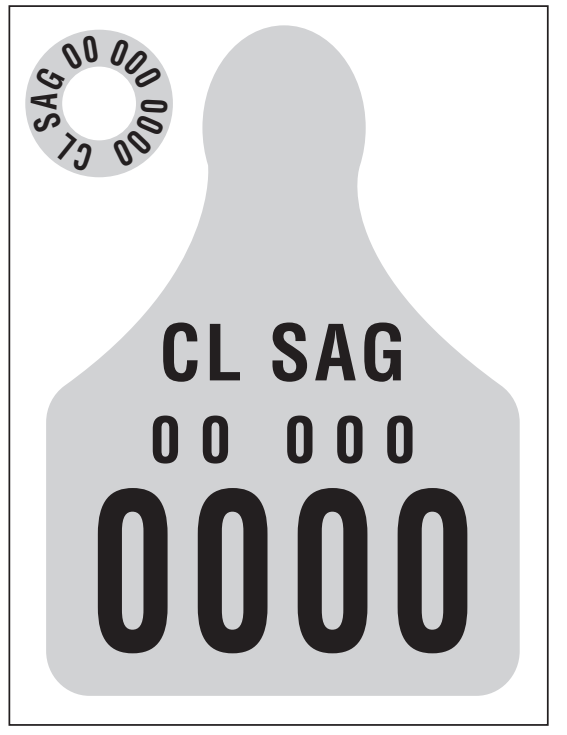

Figura 1. Modelo de dispositivo de identificación oficial (DIIO) implementado en Chile.

Official ear tag identification system model (DIIO) implemented in Chile.

dos a distancia por unidades de lectura llamadas transceivers o lectores (figura $2 \mathrm{~B}$ ). El término transponder deriva de las palabras inglesas transmit (transmisión) y respond (respuesta) y define a los dispositivos electrónicos capaces de almacenar y posteriormente transmitir una información o código que servirá para identificar de manera individual a un animal portador del mismo. Los elementos que constituyen un transponder son un circuito electrónico integrado conectado a un chip de silicio (donde se graba la información), un condensador (almacenador de energía) y una antena compuesta de una bobina de cobre sobre un núcleo de ferrita para aumentar su eficacia (Conill y col 1996, Caja y col 1998, Caja y col 2000, Ribó y col 2001). Los transponders se pueden colocar en el exterior o en el interior de los animales. Los exteriores, en general, están incluidos en material plástico protector para ser aplicados como crotales en las orejas de los animales mediante un mecanismo especial de fijación (figura 2 C). También existen los discos, medallas y hebillas que están recubiertas de material plástico y que se colocan en las extremidades y cuello de los animales mediante un dispositivo de fijación. Los internos pueden ser inyectados directamente bajo la piel de los animales, o bien, ser administrados por vía oral protegidos por un bolo. Los bolos son cápsulas de cerámica (figura 2 D) o plástico-metal, de elevado peso y que se administran oralmente quedando en forma permanente en los preestómagos de los rumiantes (figura $2 \mathrm{E}$ ). Los lectores son equipos electrónicos de mayor complejidad cuyo núcleo fundamental es un módulo de radiofrecuencia encargado de la emisión, recepción e interpretación de una señal de radiofrecuencia con la ayuda de una 


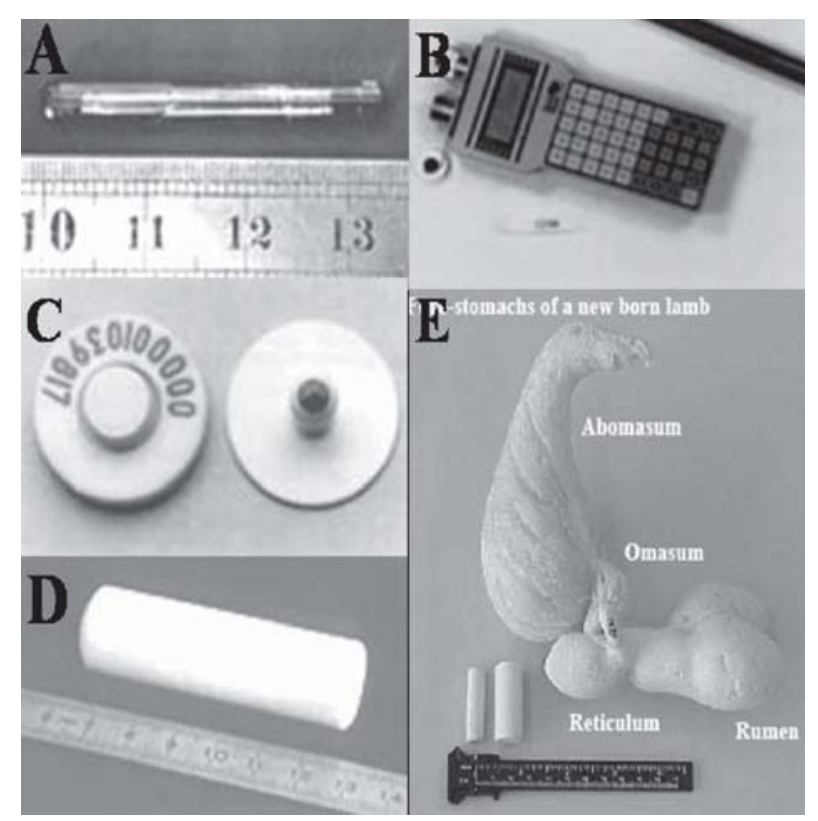

Figura 2. Sistemas de identificación electrónica (IDE) empleados en animales.

A: transponder; B: receptor; C: chip electrónico en crotal; D: bolo ruminal; E: imagen comparativa de un bolo ruminal con el estómago de un rumiante.

Electronic identification systems for animals

A: transponder; B: receptor; C: ear-tag with electronic chip; D: ruminal bolus; E: comparative image of a ruminal bolus against the stomach of a ruminant animal.

antena. Los requisitos fundamentales que debe tener un sistema de IDE son poder leerlos a distancia y con animales en movimiento, que funcionen sin pilas, que sean de larga duración y seguros para animales y para el hombre, que usen una señal codificada que pueda ser procesada por computador en forma automática, que tengan un bajo nivel de errores de identificación y de lectura, que resistan las condiciones ambientales en las que se desenvuelven los animales y que tengan un costo razonable (Caja y col 1998). Debido a la posibilidad de pérdida, de daño o de ser removidos, el bolo electrónico ruminal es el que ha adquirido mayor relevancia y el que ha demostrado mejor desempeño, pues presenta una elevada retención en el animal, no muestra fallas ni roturas, es más difícil su alteración o fraude, y es fácilmente administrable y recuperable en el matadero (Conill y col 2000; Conill 2002). Además, es inocuo y seguro para el animal, pues no se han observado efectos negativos sobre la salud ni el rendimiento productivo de estos, así como tampoco se han observado efectos negativos en la ingestión y digestibilidad de los alimentos (Caja y col 1998, Caja y col 1999). Actualmente, la Unión Europea ha aprobado el uso de sistemas de IDE para ovinos y caprinos, mientras que para bovinos se encuentra aún en discusión (Caja 2004 comunicación personal).
1.2 METOdOS BIOMETRICOS DE IDENTIFICACION ANIMAL. Los avances recientes en el desarrollo de tecnologías biométricas prometen una solución no-invasiva para la identificación individual de animales. Uno de los primeros métodos propuestos, las huellas nasales, no tuvo buena aceptación, debido principalmente a los desafíos técnicos que involucraba la obtención de una buena muestra nasal. En cambio, otros métodos se perfilan con mejores ventajas comparativas.

Reconocimiento de iris. Este sistema fue desarrollado originalmente para su uso comercial en humanos y ha sido también evaluado en animales, específicamente en caballos de carrera (Masahiko y col 2001). El proceso involucra la captura de una imagen digital del ojo. El perfil del iris es extraído y codificado para ser almacenado en una base de datos que es utilizada para reconocimiento cuando el iris es presentado para comparación. Las imperfecciones granulares en la periferia de la pupila proporcionan diferencias únicas para cada ojo. Aunque el método de escáner del iris es rápido, su uso en la identificación animal está limitado debido a que el perfil del iris no se estabiliza hasta varios meses después del nacimiento del animal y puede sufrir alteraciones debido a accidentes e infecciones.

Reconocimiento de retina. Un método alternativo ha sido desarrollado por la empresa OptiBrand (http:// www.optibrand.com), cuyo sistema de identificación y verificación animal está basado en la característica única e irrepetible del sistema vascular de la retina, el cual se presenta al nacimiento, y no cambia durante la vida del animal. De esta forma, las imágenes retinales son adquiridas a través de la pupila utilizando un computador en combinación con una cámara ocular digital. La cámara digital está conectada a un receptor interno de posicionamiento global (GPS), el cual posibilita la encriptación automática de la fecha, hora y lugar de la imagen capturada, haciendo a este sistema virtualmente imposible de violar. Además, este método permite el ingreso de información adicional como el número de crotal, códigos de tratamientos, códigos de barra de envases de vacunas y medicamentos, etc. Toda la información generada es almacenada en tarjetas de memoria removibles y los datos pueden ser recopilados rápidamente en cualquier etapa de la cadena de producción.

Huella genética del ADN (DNA fingerprinting). El principio de la identificación genética animal se basa en que, a excepción de los gemelos monocigóticos y de los clones, todos los individuos de una población animal difieren entre sí a nivel de ADN (Cunningham y Meghen 2001). De esta forma, es factible utilizar una prueba de ADN que utiliza marcadores moleculares conocidos como microsatélites para establecer estas diferencias (Lodish y col 1995). Los microsatélites, también llamados STR 
("short tandem repeats") son repeticiones muy pequeñas, las cuales proporcionan gran información sobre la diversidad y variabilidad genética de las poblaciones gracias a tres características fundamentales: i) segregan siguiendo un patrón de herencia mendeliana; ii) se encuentran distribuidos en todo el genoma, y iii) se componen de unidades repetitivas conservadas, generalmente de 2 a 6 pares de bases, pero que varían en el número de repeticiones (Zamorano y col 1998). Como consecuencia de esto último, en el largo promedio de una secuencia de un microsatélite (100-250 pares de bases) puede existir una gran diversidad de tamaños, pudiendo detectarse estas diferencias mediante las técnicas de biología molecular actualmente disponibles. Hasta el momento, los microsatélites aparecen como la más poderosa herramienta de discriminación genética entre animales (Blott y col 1999) y la Sociedad Internacional de Genética Animal (ISAG) ha recopilado y recomendado a aquellos microsatélites que proporcionan la mayor información y que sirven como marcadores estándares para la comparación entre distintas especies.

\section{TRAZABILIDAD ANIMAL COMO RESPUESTA A LOS PROBLEMAS DE SEGURIDAD ALIMENTARIA}

Si bien la trazabilidad como un nuevo concepto de seguridad alimentaria surge con mayor fuerza en la Unión Europea a raíz de la aparición de enfermedades de preocupación mundial, desde hace 15 años que en el Reino Unido existen sistemas de Aseguramiento de Calidad Predial (ACP) con el objetivo de asegurar a la industria y los consumidores que la carne fue producida en condiciones seguras, teniendo en cuenta el bienestar animal y la protección del medio ambiente. De hecho, se estima que ya al año 2002, el $75 \%$ de los novillos que llegaban a faena en el Reino Unido estaba bajo algún sistema de ACP. Estos métodos manejan normas básicas que deben dar garantía a eslabones superiores de la industria de la carne y consideran: seguridad alimentaria, identificación y trazabilidad, composición de dietas, almacenaje y uso, bienestar animal, manejo del ganado, condiciones de estabulación y manejo, protección del medio ambiente y manejo de la higiene y sanidad animal en un marco medioambiental. (MLC 2002). Los sistemas ACP mantienen como características, además de las normas o estándares mencionados, tener inspección o auditoría independiente y una implementación reconocida por los servicios de acreditación en el país o regulaciones que rigen en la Unión Europea. Si bien estos sistemas operan principalmente en el sector primario, ellos son integrados al resto de la cadena alimenticia en los pasos posteriores del producto.

En el año 1994 las directivas de la UE fueron revisadas y actualizadas para bovinos y cerdos; una de las últimas revisiones se realizó en 1997, donde se establece que cada uno de los 15 países miembros debe desarrollar bases de datos para las especies bovina y porcina, uno de cuyos resultados más importantes es el sistema de trazabilidad del ganado, con normas exactas para establecer la identificación de los animales y cómo se deben etiquetar la carne y sus productos (Pauw 2003). Más recientemente, en el llamado "Libro Blanco" sobre seguridad alimentaria de la Comisión Europea, adoptado en enero de 2000, se concretaron los principios que deben regir en la legislación alimentaria de la Unión Europea para lograr los elevados niveles de seguridad y protección que los consumidores requerían. El Libro Blanco propone un enfoque integral, "de la granja al consumidor", asumiendo que la seguridad de los alimentos comienza por los animales y los alimentos que estos consumen. También recoge la necesidad de establecer un sistema de trazabilidad. En enero de 2002, se publicó el reglamento por el que se establecen los principios y requisitos generales de la legislación alimentaria, se crea la Autoridad Europea de Seguridad Alimentaria y se fijan procedimientos relativos a la seguridad alimentaria. El Reglamento 1760/2000 de la Unión Europea estableció un sistema para la identificación y registro de los animales bovinos y para el etiquetado del bovino y productos del mismo. En este sistema se exigía que todo el vacuno y carnes frescas o congeladas sean etiquetados con un código de referencia que vincule la carne a los animales de origen, el país de sacrificio y de desposte, y los números de aprobación de los mataderos y las plantas de desposte. Complementario a esto se implementó una base de datos informática que estuvo operativa hacia fines de 1999. Desde enero de 2002 entró en vigencia la segunda fase de la normativa, la cual exigía que el etiquetado proporcionara información del país de nacimiento del animal y de todos los países donde éste había vivido. Además, se comenzaron a exigir registros actualizados en los predios de vacunas, alimentación, enfermedades, etc. (Ammendrup y Fussel 2001).

Aunque originalmente la legislación europea se enfocó al ganado vacuno, actualmente también se está dando atención a otros sectores, como el de los pequeños rumiantes, debido a los riesgos de transmisión de encefalopatía espongiforme ("scrapie"). De esta forma, están bajo consideración medidas que buscan el fortalecimiento de los requerimientos de identificación y registro de ovejas y cabras. En octubre de 2001, el Comité Científico de la Unión Europea estableció la necesidad de certificar los programas de ovinos junto con la necesidad de implementar mejores sistemas de identificación individual de esta especie animal. Es así como surge el Reglamento (CE) No 21/2004 del Consejo de 17 de diciembre de 2003, por el que se establece un sistema de identificación y registro de los animales de las especies ovina y caprina, y donde se aprueba la utilización de un sistema de identificación electrónica (IDE) para estas especies. En relación a los cerdos, en varios países de la Unión Europea, como Alemania, España y especialmen- 
te el Reino Unido, la trazabilidad e identificación se hacen por lote, no por individuo, sistema que también se ha adoptado en Chile y en Argentina (Guerra 2003). Este sistema busca llegar desde el productor final hasta el origen a nivel de desposte después de la faena, donde cada lote de desposte se compone sólo por aquel grupo de animales correspondiente a un predio o núcleo, lo que permitiría hacer un mejor seguimiento de los animales y sus productos.

\section{TRAZABILIDAD EMPLEANDO LOS METODOS DE IDENTIFICACION ANIMAL}

Como se señaló en la Introducción, se entiende por trazabilidad al seguimiento de un alimento desde su origen hasta que llega al consumidor. En el caso particular de la carne, esto significa seguir este producto en toda la cadena, desde el nacimiento del animal en el predio, pasando por el tipo de crianza y prácticas ganaderas a que fue sometido, transporte, faenado en matadero, hasta su puesta a la venta al detalle. En todo este proceso, se debe asegurar en un $100 \%$ la identidad y calidad del producto, y que ante cualquier duda o problema se pueda rastrear su paso por la cadena productiva hasta el mismo predio donde nació el animal, verificando dónde y cómo fue faenado, la forma en que fue alimentado, qué vacunas recibió, etc. Para este objetivo se emplean algunos de los sistemas de identificación animal descritos anteriormente.

3.1 TRAZABILIDAD BASADA EN IDENTIFICACION ANIMAL NO BIOMETRICA Y SU IMPLEMENTACION EN CHILE. En el caso de los sistemas de identificación no biométricos, lo usual es asignar una clave personal a cada animal (una especie de "carnet de identidad" bovino), la cual es usada para el posterior rastreo de animales. En el caso chileno, el Servicio Agrícola y Ganadero (SAG) ha comenzado la implementación del Programa Oficial de Trazabilidad Sanitaria en el rubro bovino y apícola, el que se extenderá posteriormente a la producción, ovina, avícola y porcina. Respecto al programa de trazabilidad bovina, este se enmarca dentro del Plan de Desarrollo Ganadero 2004, impulsado por el Ministerio de Agricultura (http:/ /www.trazabilidad.sag.gob.cl), y que comenzó su funcionamiento en forma oficial en noviembre de 2004, a través de cinco componentes que incluyen: i) el registro de establecimientos pecuarios bovinos; ii) el registro, aplicación y uso del dispositivo de identificación individual oficial (DIIO); iii) el registro de movimiento de bovinos; iv) la lista de medios de transporte de bovinos; v) un sistema único y oficial de información pecuaria nacional, accesible vía internet. De esta forma, desde el 1 de noviembre de 2004 se estableció un plan piloto en el que los titulares de establecimientos de la XI Región comenzaron a inscribir sus Establecimientos Pecuarios Bovinos, plan que el 1 de enero de 2005 se hizo extensi- vo al resto de las explotaciones. Todos los establecimientos involucrados recibieron un número único nacional o Rol Único Pecuario (RUP) que los identificará para todo efecto ante el SAG. Anualmente, todos estos predios deberán realizar una declaración de existencia de sus animales. Esto incluye a las ferias, mataderos, Planteles Animales Bajo Control Oficial (PABCO), según las necesidades de los respectivos programas, planteles que utilicen campos de pastoreo cordillerano o que se localicen en zonas limítrofes, y establecimientos que participan en programas de control y erradicación de enfermedades. Un componente fundamental de este Programa es la implementación de un Dispositivo de Identificación Individual Oficial (DIIO), correspondiente a un crotal o arete de color amarillo que otorgará un número único correlativo nacional a cada cabeza de ganado. Los titulares de los animales deben costear la adquisición de los dispositivos a empresas proveedoras habilitadas por el SAG. Su utilización es obligatoria para los establecimientos PABCO A, donde deberán tener crotales todos los terneros que nazcan en la presente temporada. También será obligatorio para planteles que utilicen campos de pastoreo cordillerano o que se localicen en zonas limítrofes. En el caso de planteles que participan de programas de control y erradicación de enfermedades, su uso será obligatorio, según lo indique el Manual de Procedimientos del programa respectivo. Están exentos de esta obligación los predios PABCO B y C, así como ferias y mataderos. El Programa también incluye el Formulario Sanitario de Movimiento Animal, en el que se deben registrar en forma obligatoria todos los movimientos de ganado entre los distintos establecimientos pecuarios bovinos, ferias y mataderos. Para este efecto, todos los medios de transporte de bovinos también están inscritos en un listado especial. Finalmente, el Programa incluye un sistema informático (SIPEC, Sistema Oficial de Información Pecuaria), el cual está aún en desarrollo, y que administrará este gran volumen de información con la idea de crear una red de redes, lo que permite garantizar el funcionamiento en términos de ingreso o captura, almacenamiento, análisis y consulta de toda la información requerida. La gran ventaja de contar con el registro y control de toda la existencia ganadera del país y sus movimientos está en que permitirá prevenir de manera eficiente y eficaz la eventual introducción de enfermedades, en cuyo caso ésta podrá ser detectada y erradicada en forma más oportuna.

3.2 TRAZABILIDAD MOLECULAR BASADA EN HUELLA GENETICA ANIMAL. Los sistemas de trazabilidad basados en identificación convencional de animales no son infalibles, debido a que son removidos del producto final (la carne) durante la matanza, pudiendo inducir a errores en la identificación. Por cierto, además, estos pueden ser adulterados. Para evitar este inconveniente, en varios países se están desarrollando sistemas de respaldo que puedan ase- 
gurar totalmente la trazabilidad del animal en cada una de las etapas de la cadena de producción, en especial luego del desposte del animal. Uno de estos sistemas en estudio corresponde al de trazabilidad molecular, basado específicamente en comparación de perfiles de microsatélites bovinos (Chávez y col 2004, Folch y col 2004, Vázquez y col 2004). La lógica de un sistema de trazabilidad molecular opera con la toma de una muestra biológica de origen de cada animal antes de que se pierda la identidad individual del mismo (ej.: antes de la pérdida de los crotales). Esta muestra, convenientemente identificada, se almacena por un período no inferior a la "vida útil" del producto carne, vale decir, hasta que el animal es consumido por completo. Posteriormente y en el eventual caso que en algún punto de la cadena (frigorífico, supermercado o consumidor) se quiera establecer el origen de un determinado corte de carne, se toma una segunda muestra del mismo (muestra problema). Tanto para la muestra de origen como para la muestra problema se determina un perfil específico de ADN. Estos perfiles son comparados entre sí, pudiendo establecerse si ambas huellas genéticas son idénticas o no. Si los perfiles son distintos, esto significa que la muestra problema analizada no proviene del animal con el que fue comparado.

Si bien esta tecnología no ha sido masivamente implementada, está siendo aplicada actualmente en algunos mercados muy exigentes, como Japón, donde existe un sistema de trazabilidad molecular para la carne de consumo interno (no así para las importaciones), y en España, donde la carne de elite denominada "Ternera Asturiana" entrega a sus consumidores un producto natural, único y con denominación de origen, y donde la trazabilidad molecular se aplica como el sistema que mejor garantiza la procedencia y autenticidad de dicho producto. En el caso chileno, y si bien no hay ninguna exigencia al respecto, actualmente se están realizando investigaciones tendientes elaborar un protocolo para una eventual futura aplicación de este tipo de trazabilidad (Chávez y col 2005).

Fundamento para la implementación de un sistema de trazabilidad molecular: ¿Por qué se usa ADN? Tal como se describió anteriormente, salvo los gemelos monocigóticos y los clones, todos los individuos de una población animal difieren entre sí a distintos niveles biológicos. En el nivel más primario, cada individuo puede ser distinto en su ADN. En un segundo nivel, ciertas secuencias de ADN codifican para la producción de proteínas particulares, las cuales pueden ser diferentes para cada individuo, como es el caso de las proteínas que determinan el tipo de sangre. Finalmente, los animales pueden variar en sus rasgos fenotípicos como tamaño, forma o color, características que en parte son determinadas por la genética, y en parte por las variables ambientales (Cunningham y Meghen 2001).
De todos estos tipos de variaciones, las del ADN presentan un especial atractivo para establecer métodos de identificación precisos. Por lo pronto, debido a que el genoma de cada animal contiene alrededor de tres billones de pares de bases (Cunningham y Meghen 2001), las posibilidades de variabilidad del ADN y, por ende, de singularidad de cada animal son enormes. Por otro lado, existen varios procesos biológicos muy conocidos que conducen y aumentan aún más la variación genética: la reproducción sexual, que mezcla los cromosomas de cada generación, proveyendo de un número casi ilimitado de variantes genéticas; los procesos de recombinación genética que pueden reemplazar secciones completas de un cromosoma por las de otro; las mutaciones genéticas, en las que unidades o secciones del ADN pueden ser reemplazadas, adicionadas o eliminadas. Todas estas variables contribuyen a que cada animal tenga una naturaleza genética única, la cual a su vez es acentuada por las presiones selectivas a las que se ven sometidos, generando mayores diferencias entre individuos y grupos. Por todo lo anterior, es el ADN la principal fuente de variación entre individuos y por ello una excelente herramienta para diseñar un programa de trazabilidad molecular.

Toma de muestra biológica. La operación del sistema de trazabilidad molecular contempla la toma inicial de una muestra biológica desde el animal, la cual será guardada y procesada para obtener el ADN que servirá de molde para establecer el patrón de polimorfismos específico de cada individuo, y una segunda muestra en algún punto de la cadena productiva (ej.: matadero, supermercado, etc.). Cualquier muestra biológica que contenga ADN del animal en cuestión es susceptible de ser usada (figura 3), aunque por la simpleza en su manejo las preferencias se inclinan hacia las muestras de pelo (folículo piloso) y en menor medida la sangre y tejido (músculo o piel). Sin perjuicio de lo anterior, en el caso de las muestras que se toman en la cadena de producción, de preferencia se usa un pequeño corte de músculo, aunque también es posible usar hueso y grasa. Con el advenimiento de la trazabilidad molecular, se han buscado formas para adaptar los requerimientos de esta tecnología a los sistemas de trazabilidad convencionales para poder, simultáneamente, fijar el dispositivo de identificación en el animal $\mathrm{y}$, al mismo tiempo, obtener una muestra de tejido del mismo sin temor a cometer errores o a perder la muestra correspondiente. Todo ello mediante un procedimiento rápido, simple y relativamente barato. Hace poco una empresa alemana desarrolló el dispositivo Biopsytec (www.biopsytec.de) para ambos propósitos en un solo paso, el cual está siendo probado a escala piloto en Europa con promisorios resultados. Este sistema se basa en un crotal, modificado (figura 4), el cual contiene una pequeña cápsula ("samplecap") que lleva impresa la misma leyenda y código de barras que aparecen en el crotal. Opuesto a la cápsula, este crotal lleva fijado en su eje 
metálico un proyectil. Al momento en que el crotal es cerrado, el proyectil es disparado a través de la oreja del animal, llevando consigo una pequeña muestra de tejido que es introducida en la cápsula junto al proyectil, el cual actúa como tapón. De esta manera, la muestra de tejido de la oreja del animal queda encapsulada y sellada evitando toda manipulación. En el momento en que el crotal es fijado a la oreja, la cápsula sellada conteniendo la muestra de tejido se separa del resto del crotal fijándose el dispositivo de identificación en la oreja del animal y obteniéndose, al mismo tiempo, la cápsula sellada e identificada que contiene la muestra de tejido del animal. La cápsula sólo puede ser abierta rompiéndola, por lo que la probabilidad de error o adulteración queda completamente descartada.

Para la toma de muestra, una vez faenado el animal, existe una versión simplificada de este sistema, que consiste sólo en la cápsula y el proyectil. En este caso la cápsula permanece sellada por una membrana protecto- ra que evita la contaminación y adulteración. Para este sistema, se requiere de una herramienta especial para poner el crotal ("tagger") de menor tamaño que los habituales. Mediante este sistema, se pueden recoger muestras de todos los animales de un predio al momento que se les pone el crotal, de manera que no se duplica el trabajo. Estas muestras pueden ser almacenadas en un pequeño espacio por largo tiempo, sin posibilidad de adulteración o contaminación, hasta el momento de hacer el análisis. Mayor información puede encontrarse en www.biopsytec.de.

Extracción de ADN desde las muestras biológicas. Para cada una de las muestras biológicas anteriormente descritas se han desarrollado protocolos de extracción de ADN (Jean-Pierre 1987, Miller y col 1988, Sambrook y col 1989, entre otros). Todos los protocolos de extracción desde pelo tienen como factor común la destrucción del folículo piloso con mezclas de detergentes y
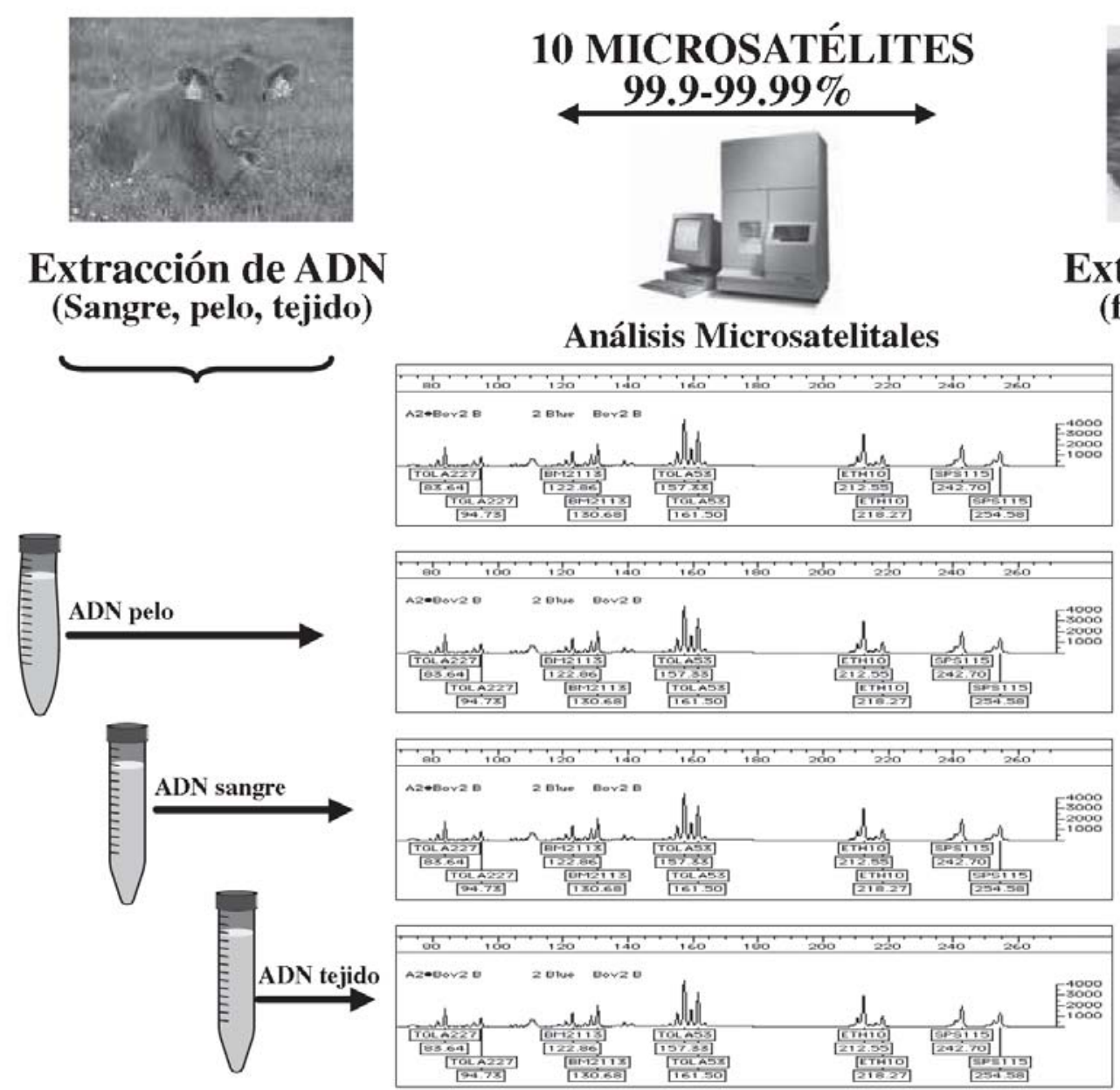

Análisis Microsatelitales

Figura 3. Sistema de trazabilidad molecular, en el cual una muestra problema es procesada y su perfil de microsatélites comparado con el perfil de microsatélites de una muestra control, que puede ser pelo, sangre o cualquier tejido del animal.

Molecular traceability system in which a sample problem is being processed and its microsatellite profile is compared with that of a tester sample which could be hair, blood or any tissue sample from the animal. 


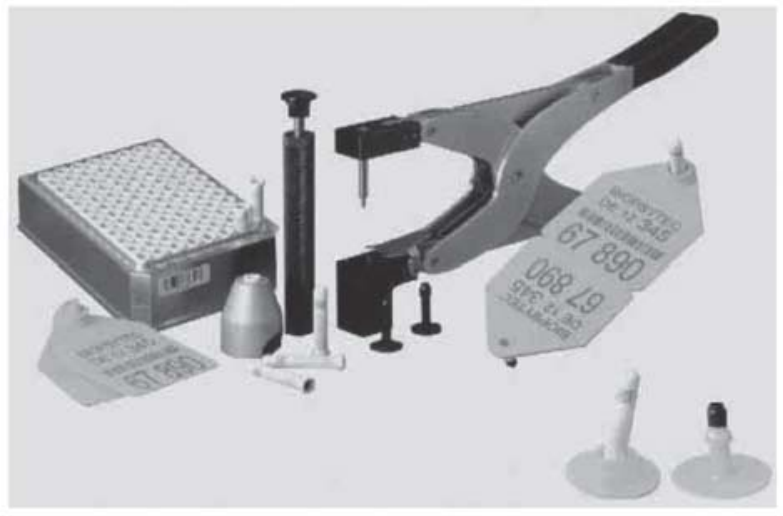

Figura 4. Sistema Biopsytec de identificación y trazabilidad animal.

Biopsytec system for animal ID and traceability.

proteasas. En algunos casos, esto puede complementarse con el uso de resinas de intercambio tipo Chelex. Sea cual sea el método, resulta bastante simple comparado con los métodos de extracción de ADN a partir de sangre o tejido. Un primer inconveniente de la sangre es su manejo, pues es necesario tomar la muestra desde el animal, procedimiento restringido solamente a médicos veterinarios en países como Inglaterra y Escocia. Además, las muestras se deben almacenar refrigeradas hasta su uso sin que coagule. En las biopsias de tejido animal, si bien se pueden usar preparados comerciales como la solución de Chomczynski, o métodos de lisis con soluciones de detergentes y proteasas, existe el inconveniente de que muchos componentes de la muestra, incluida la hemoglobina, inactivan las polimerasas necesarias para la amplificación de los microsatélites (ver más adelante). Por esta razón, se debe tener especial cuidado en la hemólisis y eliminación de estos interferentes.

Amplificación y detección de microsatélites. Para la detección de los microsatélites se utiliza un procedimiento de amplificación de ADN conocido como la reacción en cadena de la polimerasa, o PCR (Mullis y col 1986). En términos simples, la técnica consiste en que, a partir de una mezcla de reacción que contiene, entre otros componentes, una polimerasa termoestable y oligonucleótidos sintéticos que flanquean una región del ADN, se puede amplificar dicha región en forma específica hasta 100 millones de veces, gracias a ciclos de distintas temperatura que separan las hebras de ADN y que hacen posible el apareamiento de los oligonucleótidos y la acción de la polimerasa (Howe 1995). Luego, para la detección y diferenciación de los microsatélites se pueden utilizar dos sistemas. El primero consiste en la detección mediante electroforesis en geles de poliacrilamida. En esta técnica, los distintos alelos de un microsatélite amplificado por PCR migran en una matriz de poliacrilamida gracias a la aplicación de un potencial eléctrico. Esta matriz es capaz de separar estos alelos por sus diferencias en tamaño, los que luego pueden ser visualizados usando, por ejemplo, una tinción con plata. De esta forma, empleando un estándar de peso molecular adecuado, se puede establecer el patrón de polimorfismos y moléculas de ADN, que permiten establecer la identidad de las muestras analizadas (Folch y col 2004).

Con la aparición de los métodos de secuenciación automática de ADN por electroforesis capilar, se desarrolló una nueva y poderosa herramienta para la identificación automática de microsatélites. Este sistema utiliza oligonucleótidos marcados con distintos compuestos químicos fluorescentes. Usando estos oligonucleótidos, mediante PCR se amplifican los microsatélites, los cuales pueden ser analizados simultáneamente por el secuenciador automático debido a que, además de discriminarlos por tamaño, este puede identificarlos por la fluorescencia que emite el fluoróforo particular que lleva cada microsatélite. De esta manera, la manualidad y lentitud del análisis por geles de poliacrilamida es reemplazada por la automatización y rapidez de la técnica de secuenciación automática por electroforesis capilar (Chávez y col 2004).

\section{CONCLUSION}

La identificación animal y los sistemas de verificación son clave para garantizar la trazabilidad del ganado y productos derivados, desde el animal de origen, a través de las distintas etapas de producción y procesamiento, hasta su consumo. Por tanto, un sistema seguro deberá cumplir con varias necesidades, que van desde la protección del consumidor de potenciales riesgos a la salud hasta la prevención y control de las enfermedades animales. Esto permitirá controlar y verificar la producción para alcanzar estándares de calidad acordes con los requerimientos globales del comercio de ganado.

\section{RESUMEN}

La identificación de animales vivos y sus productos a través del marcaje se remonta a más de 2.000 años atrás, procedimientos que se hicieron extensivos en Europa con la aparición de las primeras epidemias. Actualmente, los avances en ingeniería genética de cultivos y animales de granja, la aparición de nuevas enfermedades relacionadas con el consumo de alimentos (ej.: mal de la vaca loca, E. coli 0157, etc.) y el descubrimiento de contaminantes en la cadena alimentaria, han provocado una mayor inquietud por parte de los consumidores. Por esta razón, garantizar la calidad del producto es un requisito fundamental para el consumidor, que exige autentificar el origen y calidad del alimento que consume. En el mercado internacional, nuevos procedimientos basados en el enfoque "de la granja a la mesa" han sido implementados en la cadena de alimentos con el objeto de resaltar marcas y procesos certificados que den garantía de calidad y seguridad alimenticia de los productos que van al mercado. Como resultado, el término "trazabilidad" de alimentos se ha ido incorporando gradualmente a nuestro vocabulario y se ha convertido en una de las principales preocupaciones de la industria alimentaria. Los sistemas de trazabilidad utilizados para identificar animales, monitorear sus 
movimientos y trazar los productos animales han evolucionado considerablemente en los últimos años. En general, estos sistemas deben ser fáciles de leer, durables e inocuos. Diferentes dispositivos han sido usados con este objetivo, tales como tatuajes, aretes, chips electrónicos, identificadores de retina e iris y, más recientemente, marcadores moleculares. Además de contar con estas herramientas, es importante armonizar los sistemas aprobados de manera de proporcionar las garantías necesarias y facilitar el comercio internacional de animales y sus productos. Hoy en día la trazabilidad es un requisito fundamental en la calidad para exportar a países más exigentes y, a la vez, una oportunidad para que el productor obtenga un producto diferenciado y a un mejor precio. Así, muchas empresas del rubro han convertido esta preocupación en una gran oportunidad comercial.

\section{AGRADECIMIENTOS}

Este trabajo fue financiado en parte por el Fondo de Desarrollo e Innovación FDI-CORFO, Proyectos 03C9AT-01 y 02C8AD-10.

\section{REFERENCIAS}

Ammendrup S, A Fussel. 2001. Legislative requirements for the identification and traceability of farm animals within the European Union. Rev Sci Tech 20, 437-44.

Blott S, J Williams, C Haley. 1999. Discriminating among cattle breeds using genetics markers. Heredity 82, 613-619.

Brappat B. 1996. Automation of weighing operations with electronic ear tags. Institut de l'Élevage, Paris, Pp 4.

Caja G, F Barillet, R Nehring, C Marie, O Ribó, E Ricard, G Lagriffoul, G Conill, M Aurel, M Jacquin. 1996. Comparison of different devices for electronic identification of dairy sheep. Producción Animal, Universidad Autónoma de Barcelona, Bellaterra and INRA Toulouse-La Fage, Castanet-Tolosan, Pp 5.

Caja G, O Ribó, R Nehring. 1998. Evaluation of migratory distance of passive transponders injected in different body sites of adult sheep for electronic identification. Livest Prod Sci 55, 279-289.

Caja G, C Conill, R Nehring, O Ribó. 1999. Development of a ceramic bolus for the permanent electronic identification of sheep, goat and cattle. Comp Electr Agric 24, 45-63.

Caja G, R Nehring, C Conill. 2000. Identifying livestock with passive transponders. Meat Automation 1, 18-21.

Chávez R, R Felmer, A Catrileo, B Sagredo, S Iraira, C Folch. 2004 Evaluación de microsatélites bovinos para la implementación de un sistema de trazabilidad molecular de la carne bovina. En: XXIX Reunión Anual de SOCHIPA, Villarrica, Chile. Libro de resúmenes Pp 151-152.

Chávez R, R Felmer, H Floody, A Catrileo, B Sagredo, S Iraira, C Folch. 2005. Implementación de un sistema de trazabilidad molecular de la carne bovina: selección de la muestra biológica definitiva y evaluación práctica en una planta de procesamiento de carne. En: XXX Reunión Anual de SOCHIPA, Temuco, Chile. Libro de resúmenes, Pp 37-38.

Commission of the European Communities. 1997. Laying down detailed rules for the implementation of Council Regulation (EC) $\mathrm{N}^{\circ} 820 / 97$ as regards eartags, holding registers and passports in the framework of the system for the identification and registration of bovine animals. Off $J$ Eur Communities 354, 19-22.

Commission of the European Communities. 2000. White Paper on food safety. Commission of the European Communities, Brussels, Pp 52.

Conill C, G Caja, R Nehring, O Ribó. 1996. Effects of injection site and transponder size in electronic identification in cattle. In: Per- formance recording of animals. State of the art, 1996. EAAP Publication $\mathrm{N}^{\circ}$ 87, Wageningen Pers, Wageningen, Pp 341-344.

Conill C, G Caja, R Nehring, O Ribó. 2000. Effects of injection position and transponder size on the performances of passive injectable transponders used for the electronic identification of cattle. J Anim Sci 78, 3001-3009.

Conill C, G Caja, R Nehring, R Ribó. 2002. The use of passive injectable transponders in fattening lambs from birth to slaughter: Effects of injection position, age, and breed. J Anim Sci 80, 919-925.

Cunningham EP, C Meghen. 2001. Biological identification systems: genetic markers. Rev Sci Tech 20, 491-499.

Folch C, B Sagredo, S Iraira, R Felmer, A Catrileo, R Chávez. 2004. Avances en el desarrollo de un método de identificación molecular para evaluar la trazabilidad en una planta de procesamiento de carne bovina de la X Región de Chile. En: XXIX Reunión Anual de SOCHIPA. Villarrica, Chile. Libro de resúmenes, Pp 153-154.

Guerra D. 2003. Experiencia en Trazabilidad: Sector Porcino. En: IICA-MINAGRI-FIA-U. de Chile (Eds.). Trazabilidad. Requisito para Carnes Exportables. Instituto Interamericano de Cooperación para la Agricultura (IICA), Subsecretaría de Agricultura Chile; Fundación para la Innovación Agraria (FIA) y Facultad de Ciencias Veterinarias y Pecuarias, Universidad de Chile. Santiago, Chile, Pp 112-122.

Howe C. 1995. Gene cloning and manipulation. Cambridge University Press. New York, N.Y.

Jean-Pierre M. 1987. A rapid method for the purification of DNA from blood. Nucleic Acids Res 15, 9611.

Lodish H, D Baltimore, A Berk, S Zipursky, P Matsudaira, J Darnell. 1995. Molecular cell biology, third edition. Scientific American Books, Inc. New York, N.Y.

Masahiko S, O Yamakita, H Shin-ichi, K Yuji, A Hiroko, S Naoki, K Ryo. 2001. A horse identification system using biometrics. Systems and Computers in Japan 32, 12-23.

MLC. 2002. Meat and Livestock Commission. Beef Yearbook 2002. Winterhill House. Snowdon Drive. Milton Keynes, UK, Pp 95.

Miller SA, D Dykes, H. Polesky. 1988. A simple salting out procedure for extracting DNA from human nucleated cells. Nucleic Acids Res $16,1215$.

Mullis K, F Faloona, S Scharf, R Saika, G Horn, H Erlich. 1986. Specific enzymatic amplification of DNA in vitro: the polymerase chain reaction. Cold Spring Harb. Symp. Quant Biol 51, 263-273.

Pauw R. 2003. Experiencias internacionales en torno al sistema de identificación y registro del ganado. En: IICA-MINAGRI-FIA-U. de Chile (Eds.). Trazabilidad. Requisito para Carnes Exportables. Instituto Interamericano de Cooperación para la Agricultura (IICA), Subsecretaría de Agricultura Chile; Fundación para la Innovación Agraria (FIA) y Facultad de Ciencias Veterinarias y Pecuarias, Universidad de Chile. Santiago, Chile, Pp 81-93.

Pettitt RG. 2001. Traceability in the food animal industry and supermarket chains. Rev Sci Tech 20, 584-597.

Ribó O, C Korn, U Meloni, M Cropper, P De Winne, M Cuype 2001. IDEA: a large-scale project on electronic identification of livestock. Rev. Sci Tech Off int Epiz 20, 426-436.

Sambrook J, E. Fritsch, T Maniatis. 1989. Molecular cloning: a laboratory manual (2nd ed.). Cold Spring Harbor Laboratory Press. Cold Spring Harbor, N.Y.

Stanford K, J Stitt, J Kellar, T McAllister. 2001.Traceability in cattle and small ruminants in Canada. Rev Sci Tech 20, 510-22.

Vázquez J, T Pérez, F Urena, E Gudin, J Albornoz, A Domínguez. 2004. Practical application of DNA fingerprinting to trace beef. J Food Prot 67, 972-9.

Zamorano M, J Ruiter, A Rodero, J Vega-pla. 1998. Análisis genético de marcadores microsatélites de la raza bovina berrenda en negro. Arch Zootec 47, 195-200. 